INS-Rep-1162

DPNU-96-50

September, 1996

\title{
A String-inspired Ansatz for Quark Masses and Mixing
}

\author{
TAtsuo Kobayashi 円 \\ Institute for Nuclear Study, University of Tokyo, Tanashi, Tokyo 188, Japan \\ ZHI-ZHONG XING \\ Department of Physics, Nagoya University, Chikusa-ku, Nagoya 464-01, Japan
}

\begin{abstract}
We propose a simple but realistic pattern of quark mass matrices at the string scale, which can be derived from orbifold models of superstring theory with no use of gauge symmetries. This pattern is left-right symmetric and preserves the structural parallelism between up and down quark sectors. Its phenomenological consequences on flavor mixing and $C P$ violation are confronted with current experiments at the weak scale by use of the renormalization-group equations in the framework of the minimal supersymmetric standard model. We find that good agreement is achievable without fine-tuning.
\end{abstract}

\footnotetext{
1 Electronic address: Kobayast@ins.u-tokyo.ac.jp

${ }^{2}$ Electronic address: Xing@eken.phys.nagoya-u.ac.jp
} 


\section{Introduction}

The origin of fermion masses is one of the most important problems in particle physics. Higher dimension couplings like $H Q q(\theta / M)^{n}$ can explain the hierarchical structure of fermion masses and flavor mixing angles [1, 2, 3]. The key point is that these couplings may provide effective Yukawa couplings with suppression factors $(\langle\theta\rangle / M)^{n}$, when suitable fields such as $\theta$ develop vacuum expectation values (VEVs). Underlying symmetries provide selection rules for higher dimension couplings as well as renormalizable couplings. In this case, the structure of powers $n$ of suppression factors are determined in terms of some types of quantum numbers from underlying symmetries.

For example, gauge symmetries including the anomalous $U(1)$ symmetry have been used to constrain such powers in nonrenormalizable couplings [1, 3]. In this case one needs different quantum numbers for families under gauge symmetries, which are broken by VEVs of scalar fields, in order to obtain a realistic hierarchy of fermion masses. On the other hand, through such symmetry breaking we have $D$-term contributions to soft scalar masses, which are proportional to quantum numbers of broken gauge symmetries [4]. Thus different quantum numbers for families naturally resolve the degeneracy of sfermion masses. However, current measurements of the flavor changing neutral current (FCNC) processes require the degeneracy of sfermion masses, at least, for the first and second families of squarks [5].

In the fermion mass generation mechanisms reviewed above, one needs the symmetries which lead to the hierarchical fermion mass matrices while preserving the required degeneracy of soft scalar masses without fine-tuning. Gauge symmetries are not such symmetries. Superstring theory is the only known candidate for a successful unified theory of all the interactions including gravity. In superstring theory, selection rules for higher dimension couplings are provided by symmetries of a six-dimensional comactified space as well as gauge symmetries. These "stringy symmetries" due to the six-dimensional compactified space have no direct relation with soft scalar masses, and the fermion mass generation due to these stringy symmetries preserves the degeneracy of sfermion masses. That is the difference between stringy symmetries and gauge symmetries. In Ref. [6], selection rules for nonrenormalizable couplings are discussed explicitly within the framework of orbifold models [7]. These selection rules can lead to realistic quark mass matrices. Actually one of simple but instructive mass matrices has been derived from a variety of orbifold models [8] [? The pattern is left-right symmetric, and a parallel structure exists between up and down quark sectors.

It seems natural that left-right symmetric mass matrices have the parallel structure for

\footnotetext{
${ }^{3}$ Note that some different attempts have been made to derive the fermion mass matrices from other types of four-dimensional string models. See, e.g., Ref. [9].
} 
up and down quark sectors in the above-mentioned fermion mass generation mechanism. The reason is quite straightforward: if quark mass matrices are left-right symmetric, they are dominated by quantum numbers of the quark doublets $Q_{i}$ and the Higgs fields. Of course, the up-down symmetric quark mass matrices can also be obtained through other approaches, e.g., by using an extra $U(1)$ symmetry [3].

In this work we want to study a simple and realistic pattern of quark mass matrices at the string scale $M_{\mathrm{st}}$, which can be derived from orbifold models of superstring theory. This type of quark mass matrices is left-right symmetric and has the structural parallelism between up and down quark sectors. By use of the renormalization-group equations in the framework of the minimal supersymmetric standard model (MSSM) below $M_{\text {st }}$, we confront the consequences of our string-inspired mass pattern with current experimental data at the weak scale $M_{Z}$. The flavor mixing matrix elements $\left|V_{u s}\right|,\left|V_{c d}\right|,\left|V_{u b} / V_{c b}\right|$ and $\left|V_{t d} / V_{t s}\right|$ can be scale-independently obtained in terms of the quark mass ratios $m_{u} / m_{c}$ and $m_{d} / m_{s}$ (as well as a $C P$-violating phase shift) in leading order approximations. So are three inner angles of the unitarity triangle $V_{u b}^{*} V_{u d}+V_{c b}^{*} V_{c d}+V_{t b}^{*} V_{t d}=0$. For reasonable values of $\tan \beta_{\text {susy }}$ (the ratio of Higgs vacuum expectation values in the MSSM), we find that the renormalized $\left|V_{c b}\right|$ and $\left|V_{t s}\right|$ at $M_{Z}$ can fit current data very well.

\section{Quark mass matrices in superstring theory}

In general, the underlying theory like supergravity or superstring theory has nonrenormalizable couplings as

$$
h_{\mathrm{u} i j} H_{2} Q_{i} u_{j}\left(\theta_{\mathrm{u}} / M_{2}\right)^{n_{i j}}, \quad h_{\mathrm{d} i j} H_{1} Q_{i} d_{j}\left(\theta_{\mathrm{d}} / M_{1}\right)^{n_{i j}^{\prime}}
$$

where $u_{j}\left(d_{j}\right)$ denotes the up type (down type) of $S U(2)$ singlet quark fields, and $H_{2,1}$ are the Higgs fields for the up and down sectors. Here $h_{\mathrm{u} i j}$ and $h_{\mathrm{d} i j}$ denote the coupling strengths, which can be calculated within the framework of superstring theory. Their magnitudes are of $O(1)$ in most cases. When the fields $\theta_{\mathrm{u}, \mathrm{d}}$ develop VEVs, these couplings become Yukawa couplings with suppression factors $\varepsilon_{\mathrm{u}}=\left(\left\langle\theta_{\mathrm{u}}\right\rangle / M_{2}\right)^{n_{i j}}$ and $\varepsilon_{\mathrm{d}}=\left(\left\langle\theta_{\mathrm{d}}\right\rangle / M_{1}\right)^{n_{i j}^{\prime}}$. These can lead to a hierarchical structure in the fermion mass matrices. Here we restrict ourselves to the case where up and down mass matrices have the parallel structure, i.e., $n_{i j}=n_{i j}^{\prime}$.

The structure of the powers $n_{i j}$ depends on underlying symmetries. If gauge symmetry breaking is dominant for the fermion mass generation, we obtain nondegenerate soft sfermion masses due to $D$-term contribution of broken symmetries in the case that fine-tuning is absent. For example, nondegenerate soft scalar masses are in general derived from supersymmetry (SUSY) breaking within the superstring framework, except the dilaton-dominant SUSY breaking case with no anomalous $U(1)$ symmetry [10]. This nondegeneracy is dangerous for the 
FCNC. We need the symmetries which constrain nonrenormalizable couplings, but do not lead to $D$-term contributions to soft scalar masses.

Superstring theory has such symmetries, i.e. stringy symmetries. The orbifold construction is one of the simplest and most interesting constructions to derive four-dimensional string vacua [0]. In orbifold models, string states consist of the bosonic string on the four-dimensional spacetime and a six-dimensional orbifold, their right-moving superpartners and left-moving gauge parts. This six-dimensional part and its supersymmetric part lead to complicated selection rules for allowed couplings [11, 12, 13]. In orbifold models each matter field corresponds to a $\theta^{k}$-twisted sector $T_{k}$ as well as the untwisted sector. Stringy symmetries constrain couplings among these sectors. Allowed nonrenormalizable couplings have been shown explicitly in Ref. [6]. Further orbifolds have some singular points, i.e., fixed points for each $T_{k}$ sector. Each matter field is assigned to one of fixed points in $T_{k}$. Stringy symmetries constrain combinations of fixed points for nonvanishing couplings [12. These selection rules for nonrenormalizable couplings cannot be understood in terms of effective field theories [13, 6]. Thus, if we assign the matter fields to these sectors and fixed points in certain way, we can obtain realistic fermion mass matrices.

Actually in Ref. [8] an instructive pattern of quark mass matrices has been obtained as

$$
M_{\mathrm{u}, \mathrm{d}}=c_{\mathrm{u}, \mathrm{d}}\left(\begin{array}{ccc}
\varepsilon_{11} & \varepsilon_{\mathrm{u}, \mathrm{d}}^{3} & \varepsilon_{13} \\
\varepsilon_{\mathrm{u}, \mathrm{d}}^{3} & \varepsilon_{\mathrm{u}, \mathrm{d}}^{2} & \varepsilon_{\mathrm{u}, \mathrm{d}}^{2} \\
\varepsilon_{13} & \varepsilon_{\mathrm{u}, \mathrm{d}}^{2} & 1
\end{array}\right),
$$

up to $h_{\mathrm{u} i j} / h_{\mathrm{u} 33}\left(h_{\mathrm{d} i j} / h_{\mathrm{d} 33}\right)$ of $O(1)$, where $c_{\mathrm{u}}=h_{\mathrm{u} 33}\left\langle H_{2}\right\rangle$ and $c_{\mathrm{d}}=h_{\mathrm{d} 33}\left\langle H_{1}\right\rangle$. For instance, this form of mass matrix is obtainable in the case where the Higgs fields are assigned to $T_{4}$ and the first, second and third families of quarks are assigned to $T_{1}, T_{2}$ and $T_{4}$ respectively, provided certain fields in $T_{1}, T_{2}$ and $T_{4}$ develop VEVs. In this treatment, the $(1,1),(1,3)$ and $(3,1)$ matrix elements do not vanish completely. However, these elements are strongly suppressed in comparison with their nearest-neighboring elements, thus they can always be taken as zero for any phenomenological purpose.

In general, we expect $\varepsilon_{\mathrm{u}} \neq \varepsilon_{\mathrm{d}}$. For example, the mixing between light and heavy Higgs fields leads to $\varepsilon_{\mathrm{u}} \neq \varepsilon_{\mathrm{d}}$ [2, 3]. The coupling strengths $h_{\mathrm{u} i j}\left(h_{\mathrm{d} i j}\right)$ are calculated as $h_{\mathrm{u} i j} \sim \exp \left(-a_{i j} T\right)$ $\left(h_{\mathrm{d} i j} \sim \exp \left(-a_{i j}^{\prime} T\right)\right)$, where $T$ is the moduli parameter representing the size of six-dimensional compactified space and $a_{i j}\left(a_{i j}^{\prime}\right)$ is a constant depending on the combination of fixed points for couplings [11] The factors $\exp \left(-a_{i j} T\right)$ in the mass matrix elements are generally different

\footnotetext{
${ }^{4}$ Similarly the $C P$-violating phases can be introduced into some elements in the case of nonvanishing background antisymmetric tensors [14], although without such antisymmetric tensors $C P$ is a nice symmetry of superstring and couplings are always real except trivial phases $[15]$.
} 
from one another. Thus we phenomenologically introduce two additional parameters $\omega_{\mathrm{u}, \mathrm{d}}$ for the $(2,2)$ elements and discuss mass matrices of the form

$$
M_{\mathrm{u}, \mathrm{d}}=c_{\mathrm{u}, \mathrm{d}}\left(\begin{array}{ccc}
0 & \varepsilon_{\mathrm{u}, \mathrm{d}}^{3} & 0 \\
\varepsilon_{\mathrm{u}, \mathrm{d}}^{3} & \omega_{\mathrm{u}, \mathrm{d}} \varepsilon_{\mathrm{u}, \mathrm{d}}^{2} & \varepsilon_{\mathrm{u}, \mathrm{d}}^{2} \\
0 & \varepsilon_{\mathrm{u}, \mathrm{d}}^{2} & 1
\end{array}\right) .
$$

Although $\omega_{\mathrm{u}, \mathrm{d}} \sim O(1)$ are taken as a phenomenological assumption, we hope that they will have a theoretical explanation, analogous to that for $\varepsilon_{\mathrm{u}, \mathrm{d}}$. In fact, we can assign fixed points for the matter fields so that such factors appear only for the $(2,2)$ matrix elements. Of course an introduction of similar factors for the other matrix elements of $M_{\mathrm{u}, \mathrm{d}}$ is not interesting in phenomenology.

Clearly the pattern of $M_{\mathrm{u}, \mathrm{d}}$ can be regarded as a non-trivial generalization of the Fritzsch Ansatz [16, 17] at the string scale $M_{\mathrm{st}}=3.7 \times 10^{17} \mathrm{GeV}$. Diagonalizing $M_{\mathrm{u}, \mathrm{d}}$ through the orthogonal transformations $O_{\mathrm{u}, \mathrm{d}}^{\mathrm{T}} M_{\mathrm{u}, \mathrm{d}} O_{\mathrm{u}, \mathrm{d}}=\operatorname{Diag}\left\{m_{u, d}, m_{c, s}, m_{t, b}\right\}$, we are able to obtain the mass eigenvalues. In lowest order approximations, we find

$$
\begin{array}{rlrl}
m_{t} & \approx c_{\mathrm{u}}, & m_{c} \approx \varepsilon_{\mathrm{u}}^{2} \omega_{\mathrm{u}} c_{\mathrm{u}}, & m_{\mathrm{u}} \approx \frac{\varepsilon_{\mathrm{u}}^{4}}{\omega_{\mathrm{u}}} c_{\mathrm{u}} ; \\
m_{b} \approx c_{\mathrm{d}}, & m_{s} \approx \varepsilon_{\mathrm{d}}^{2} \omega_{\mathrm{d}} c_{\mathrm{d}}, & m_{d} \approx \frac{\varepsilon_{\mathrm{d}}^{4}}{\omega_{\mathrm{d}}} c_{\mathrm{d}} .
\end{array}
$$

These lead to the following quark mass relations:

$$
\frac{\omega_{\mathrm{u}}^{2} m_{u}}{m_{c}} \approx \frac{m_{c}}{\omega_{\mathrm{u}} m_{t}} \approx \varepsilon_{\mathrm{u}}^{2}, \quad \frac{\omega_{\mathrm{d}}^{2} m_{d}}{m_{s}} \approx \frac{m_{s}}{\omega_{\mathrm{d}} m_{b}} \approx \varepsilon_{\mathrm{d}}^{2} .
$$

Although simpler and more instructive geometrical relations for $m_{u}, m_{c}, m_{t}$ and $m_{d}, m_{s}, m_{b}$ can be respectively obtained if one takes $\omega_{\mathrm{u}}=\omega_{\mathrm{d}}=1$, the latter will not be favored by current data at (or below) the weak scale $M_{Z}=91.187 \mathrm{GeV}$. This is a phenomenological reason for the essential presence of free parameters $\omega_{\mathrm{u}, \mathrm{d}}$ in $M_{\mathrm{u}, \mathrm{d}}$.

To calculate the mixing matrix of quark flavors, we need introduce a phase matrix $P=$ $\operatorname{Diag}\left\{1, e^{\mathrm{i} \phi}, e^{\mathrm{i} \phi}\right\}$, where $\phi$ denotes the possible phase difference between $M_{\mathrm{u}}$ and $M_{\mathrm{d}}$. Such a $C P$-violating phase may arise from the dynamical details of our fermion mass generation mechanism, e.g., the background antisymmetric tensors in orbifold models or imaginary VEVs of $\theta$. Phenomenologically the existence of $\phi$ is necessary for the Ansatz to properly reproduce the Cabibbo angle and $C P$ violation. The flavor mixing matrix, defined as $V \equiv O_{\mathrm{u}}^{\mathrm{T}} P O_{\mathrm{d}}$, takes the following form in leading order approximations:

$$
V \approx\left(\begin{array}{ccc}
1-\frac{1}{2} \varepsilon_{\mathrm{d}}^{\prime 2} & \varepsilon_{\mathrm{u}}^{\prime} e^{\mathrm{i} \phi}-\varepsilon_{\mathrm{d}}^{\prime} & \varepsilon_{\mathrm{u}}^{\prime}\left(\varepsilon_{\mathrm{d}}^{2}-\varepsilon_{\mathrm{u}}^{2}\right) e^{\mathrm{i} \phi} \\
\varepsilon_{\mathrm{d}}^{\prime} e^{\mathrm{i} \phi}-\varepsilon_{\mathrm{u}}^{\prime} & \left(1-\frac{1}{2} \varepsilon_{\mathrm{d}}^{\prime 2}\right) e^{\mathrm{i} \phi} & \left(\varepsilon_{\mathrm{d}}^{2}-\varepsilon_{\mathrm{u}}^{2}\right) e^{\mathrm{i} \phi} \\
\varepsilon_{\mathrm{d}}^{\prime}\left(\varepsilon_{\mathrm{u}}^{2}-\varepsilon_{\mathrm{d}}^{2}\right) e^{\mathrm{i} \phi} & \left(\varepsilon_{\mathrm{u}}^{2}-\varepsilon_{\mathrm{d}}^{2}\right) e^{\mathrm{i} \phi} & e^{\mathrm{i} \phi}
\end{array}\right),
$$


where $\varepsilon_{\mathrm{u}, \mathrm{d}}^{\prime}=\varepsilon_{\mathrm{u}, \mathrm{d}} / \omega_{\mathrm{u}, \mathrm{d}}$. This result will be confronted with current experimental data at the weak scale $M_{Z}$ in the next section.

\section{Quark mixing and $C P$ violation at the weak scale}

We are in a position to compare the flavor mixing matrix $V$ with low-energy data, so as to phenomenologically justify the string-inspired quark mass matrices $M_{\mathrm{u}, \mathrm{d}}$. For this purpose, one has to run the results obtained at the string scale $M_{\text {st }}$ to the weak scale $M_{Z}$. We assume the MSSM for spontaneous symmetry breaking below $M_{\mathrm{st}}$, and then make use of the corresponding renormalization-group equations for quark mass matrices and $V$.

The one-loop renormalization group equations for quark mass ratios and flavor mixing matrix elements have been explicitly presented by Babu and Shafi in Ref. [18. In view of the hierarchy of Yukawa couplings and quark mixing angles, one can make reliable analytical approximations for the relevant evolution equations by keeping only the leading terms. It has been found that (1) the running effects of $m_{u} / m_{c}$ and $m_{d} / m_{s}$ are negligibly small; (2) the diagonal elements of the flavor mixing matrix have negligible evolutions with energy; (3) the evolutions of $\left|V_{u s}\right|$ and $\left|V_{c d}\right|$ involve the second-family Yukawa couplings and thus they are negligible; (4) the flavor mixing matrix elements $\left|V_{u b}\right|,\left|V_{c b}\right|,\left|V_{t d}\right|$ and $\left|V_{t s}\right|$ have identical running behaviors. Taking these points into account, we first notice that $\varepsilon_{\mathrm{u}}^{\prime} \approx\left(m_{u} / m_{c}\right)^{1 / 2}$ and $\varepsilon_{\mathrm{d}}^{\prime} \approx\left(m_{d} / m_{s}\right)^{1 / 2}$ are approximately scale-independent. Consequently some scale-independent results for quark mixings at $M_{Z}$ can be straightforwardly obtained as follows.

(a) The magnitudes of three diagonal elements of $V$ are close to unity, i.e., $\left|V_{u d}\right| \approx\left|V_{c s}\right| \approx$ $\left|V_{t b}\right| \approx 1$. A more careful estimate with the help of unitarity of $V$ leads to the fine hierarchy $\left|V_{t b}\right|>\left|V_{u d}\right|>\left|V_{c s}\right|$. This result is consistent with current data 19].

(b) The flavor mixing matrix elements $\left|V_{u s}\right|$ and $\left|V_{c d}\right|$ in the leading order approximation read

$$
\left|V_{u s}\right| \approx\left|V_{c d}\right| \approx\left[\frac{m_{u}}{m_{c}}+\frac{m_{d}}{m_{s}}-2\left(\frac{m_{u} m_{d}}{m_{c} m_{s}}\right)^{1 / 2} \cos \phi\right]^{1 / 2}
$$

Since $\left|V_{u s}\right|\left(=0.2205 \pm 0.0018\right.$ [20]) has been accurately measured and a reliable value for $m_{s} / m_{d}$ $(=18.9 \pm 0.8$ [21]) has been obtained from chiral perturbation theory, we are able to constrain the phase shift $\phi$ in spite of the large uncertainty associated with $m_{u} / m_{c}\left(\sim 5 \times 10^{-3}[20\right.$ ]). We find $73^{0} \leq \phi \leq 82^{0}$ only if $m_{u} / m_{c} \geq 10^{-3}$.

(c) The ratios $\left|V_{u b} / V_{c b}\right|$ and $\left|V_{t d} / V_{t s}\right|$ are obtained as

$$
\left|\frac{V_{u b}}{V_{c b}}\right| \approx\left(\frac{m_{u}}{m_{c}}\right)^{1 / 2}, \quad\left|\frac{V_{t d}}{V_{t s}}\right| \approx\left(\frac{m_{d}}{m_{s}}\right)^{1 / 2}
$$


to a good degree of accuracy. By use of $m_{s} / m_{d}=18.9 \pm 0.8$ [21], we get $0.225 \leq\left|V_{t d} / V_{t s}\right| \leq$ 0.235. This result is consistent with that extracted from the analysis of current experimental data [22]: $0.15 \leq\left|V_{t d} / V_{t s}\right| \leq 0.34$. On the other hand, the allowed region of $\left|V_{u b} / V_{c b}\right|$ is restricted by that of $m_{u} / m_{c}$, which has not been reliably determined. We find that $0.0036 \leq$ $m_{u} / m_{c} \leq 0.01$ is required by our Ansatz in fitting the data $\left|V_{u b} / V_{c b}\right|=0.08 \pm 0.02$ [20].

(d) The unitarity triangle $V_{u b}^{*} V_{u d}+V_{c b}^{*} V_{c d}+V_{t b}^{*} V_{t d}=0$ can be approximately derived from our quark mass Ansatz. From Eqs. (6) and (7) we observe that $V_{c d},\left(m_{u} / m_{c}\right)^{1 / 2}$ and $\left(m_{d} / m_{s}\right)^{1 / 2}$ form a triangle in the complex plane [17]. Rescaling three sides of this triangle by $V_{c b}^{*}$ and making use of Eq. (8), we then reproduce the above-mentioned unitarity triangle in leading order approximations. Thus three inner angles of the unitarity triangle, commonly denoted as $\alpha, \beta$ and $\gamma$ [20], can be calculated in terms of quark mass ratios and $\phi$. In $B$-meson physics, one is more interested in the characteristic $C P$-violating observables $\sin (2 \alpha), \sin (2 \beta)$ and $\sin \gamma$, which will be detected at the forthcoming $B$ factories. Explicitly, we find

$$
\begin{aligned}
\sin (2 \alpha) & \approx \sin (2 \phi) \\
\sin (2 \beta) & \approx \frac{2 \sin \phi(r-\cos \phi)}{1-2 r \cos \phi+r^{2}} \\
\sin ^{2} \gamma & \approx \frac{r^{2} \sin ^{2} \phi}{1-2 r \cos \phi+r^{2}}
\end{aligned}
$$

where $r=\left[\left(m_{c} m_{d}\right) /\left(m_{u} m_{s}\right)\right]^{1 / 2}$. A brief estimate gives $0.18 \leq \sin (2 \alpha) \leq 0.58,0.5 \leq \sin (2 \beta) \leq$ 0.78 and $0.93 \leq \sin ^{2} \gamma \leq 1.0$, which are very restrictive but consistent with current data analyzed by Ali and London [22]: $-0.90 \leq \sin (2 \alpha) \leq 1.0,0.32 \leq \sin (2 \beta) \leq 0.94$ and $0.34 \leq$ $\sin ^{2} \gamma \leq 1.0$

We want to stress that all the scale-independent results obtained above are indeed the consequences of the Fritzsch Ansatz [16]. They appear naturally only if the $(1,1),(1,3)$ and $(3,1)$ elements of $M_{\mathrm{u}, \mathrm{d}}$ are greatly suppressed in comparison with their nearest-neighboring elements [23, 24]. Next we discuss the scale dependence of $\left|V_{c b}\right|$ and $\left|V_{t s}\right|$, whose magnitudes in our Ansatz are absolutely different from those in the Fritzsch Ansatz or other quark mass patterns [25, 26].

Non-negligible running effects from the string scale $M_{\text {st }}$ to the weak scale $M_{Z}$ can manifest themselves in the expressions of $\left|V_{c b}\right|$ and $\left|V_{t s}\right|$, which depend strongly upon the mass ratios $m_{c} / m_{t}$ and $m_{s} / m_{b}$. The evolution functions relevant to the calculation of $\left|V_{c b}\right|$ or $\left|V_{t s}\right|$ are defined as

$$
\xi_{t, b}=\exp \left[-\frac{1}{16 \pi^{2}} \int_{0}^{\ln \left(M_{\mathrm{st}} / M_{Z}\right)} f_{t, b}^{2}(\chi) \mathrm{d} \chi\right],
$$

where $\chi \equiv \ln \left(\mu / M_{Z}\right), f_{t}$ and $f_{b}$ are the respective Yukawa coupling eigenvalues of the top and bottom quarks. A good approximation is that the third-family Yukawa couplings of 
quarks and charged leptons, together with the gauge couplings, play the dominant roles in the renormalization-group equations [18]. Then the magnitudes of $\xi_{t}$ and $\xi_{b}$ can be evaluated for arbitrary $\tan \beta_{\text {susy }}$ from $M_{\text {st }}$ to $M_{Z}$, as done in 25] with the typical inputs $m_{t}\left(M_{Z}\right) \approx 180$ $\mathrm{GeV}, m_{b}\left(M_{Z}\right) \approx 3.1 \mathrm{GeV}$ and $m_{\tau}\left(M_{Z}\right) \approx 1.78 \mathrm{GeV}$. For our present purpose, the numerical results of $\xi_{t}$ and $\xi_{b}$ as functions of $\tan \beta_{\text {susy }}$ are illustrated in Fig. 1. Three key evolution relations in the MSSM are given as follows 25]:

$$
\begin{aligned}
\left.\frac{m_{s}}{m_{b}}\right|_{M_{Z}} & =\left.\frac{1}{\xi_{t} \xi_{b}^{3}} \frac{m_{s}}{m_{b}}\right|_{M_{\mathrm{st}}}, \\
\left.\frac{m_{c}}{m_{t}}\right|_{M_{Z}} & =\left.\frac{1}{\xi_{t}^{3} \xi_{b}} \frac{m_{c}}{m_{t}}\right|_{M_{\mathrm{st}}}, \\
\left|\hat{V}_{i j}\right|_{M_{Z}} & =\frac{1}{\xi_{t} \xi_{b}}\left|\hat{V}_{i j}\right|_{M_{\mathrm{st}}},
\end{aligned}
$$

where $(i j)=(u b),(c b),(t d)$ or $(t s)$. With the help of Eq. (6), we obtain the renormalized $\left|V_{c b}\right|$ and $\left|V_{t s}\right|$ at $M_{Z}$ in leading order approximations:

$$
\left|V_{c b}\right| \approx\left|V_{t s}\right| \approx \frac{\xi_{b}}{\xi_{t}^{1 / 3}}\left(\frac{m_{d}}{m_{s}}\right)^{1 / 3}\left(\frac{m_{s}}{m_{b}}\right)^{2 / 3}-\frac{\xi_{t}}{\xi_{b}^{1 / 3}}\left(\frac{m_{u}}{m_{c}}\right)^{1 / 3}\left(\frac{m_{c}}{m_{t}}\right)^{2 / 3}
$$

This instructive result is a unique consequence of our quark mass Ansatz given in Eq. (3). Typically taking $m_{u} / m_{c}=0.006, m_{c} / m_{t}=0.005, m_{d} / m_{s}=0.051 \sim 0.055$ and $m_{s} / m_{b}=0.03 \sim$ 0.04 [21, 27], we confront Eq. (12) with the experimental data on $V_{c b}$ (i.e., $\left|V_{c b}\right|=0.0388 \pm 0.0032$ [28]). As shown in Fig. 2, our result is in good agreement with experiments for $\tan \beta_{\text {susy }}<55$. This implies that the quark mass matrices $M_{\mathrm{u}, \mathrm{d}}$, proposed at the string scale $M_{\mathrm{st}}$, may have a large chance to survive for reasonable values of $\tan \beta_{\text {susy }}$.

\section{Summary}

We have derived a simple and realistic pattern of quark mass matrices from orbifold models of superstring theory at the string scale $M_{\mathrm{st}}$. It is worth emphasizing that gauge symmetries are not needed for our purpose. This point is so important that one can preserve the degeneracy of squark masses without fine-tuning. The obtained up and down mass matrices have the parallel structure, and each of them are left-right symmetric. They totally consist of seven free parameters, thus can lead to three independent predictions for flavor mixing angles at the string scale. From the purely phenomenological point of view, our quark mass pattern can be regarded as a non-trivial generalization of the Fritzsch Ansatz.

The consequences of our string-inspired mass matrices $M_{\mathrm{u}, \mathrm{d}}$ on flavor mixing and $C P$ violation have been confronted with current experimental data at the weak scale $M_{Z}$ by use of the one-loop renormalization-group equations in the MSSM framework. In leading order approximations, we find that $\left|V_{u s}\right|,\left|V_{c d}\right|,\left|V_{u b} / V_{c b}\right|$ and $\left|V_{t d} / V_{t s}\right|$ are scale-independent. Consequently 
three inner angles of the unitarity triangle $V_{u b}^{*} V_{u d}+V_{c b}^{*} V_{c d}+V_{t b}^{*} V_{t d}=0$ are approximately scaleindependent, and their magnitudes are independent of $m_{c} / m_{t}$ and $m_{b} / m_{t}$ to a good degree of accuracy. We get very restrictive results $0.18 \leq \sin (2 \alpha) \leq 0.58,0.5 \leq \sin (2 \beta) \leq 0.78$ and $0.93 \leq \sin ^{2} \gamma \leq 1.0$ (see also Ref. [25]), which can be tested in the forthcoming experiments of $B$-meson factories.

The flavor mixing matrix elements, which are most sensitive to the features of different quark mass Ansätze, are usually $\left|V_{c b}\right|$ and $\left|V_{t s}\right|$. Our mass pattern yields a unique expression for $\left|V_{c b}\right|$ (or $\left|V_{t s}\right|$ ) in terms of quark mass ratios $m_{u} / m_{c}, m_{c} / m_{t}, m_{d} / m_{s}$ and $m_{s} / m_{b}$. At the weak scale $M_{Z}$, the renormalized $\left|V_{c b}\right|$ can fit current data very well for reasonable values of $\tan \beta_{\text {susy }}$. Of course, all numerical results depend upon the inputs of quark mass eigenvalues, which still have large uncertainties.

The good agreement between the string-inspired mass pattern and current data implies that the former may have a large chance to be true. To reduce the number of free parameters in our Ansatz, a possible way is to relate the quark mass matrices with the lepton mass matrices at the string scale. Then one should be able to predict the quark masses in terms of the lepton masses with the help of some discrete symmetries (see, e.g., Ref. [29]). Such possibilities are of course attractive from both theoretical and phenomenological viewpoints, and they will be studied elsewhere.

\section{Acknowledgements}

One of the authors (ZZX) would like to thank A.I. Sanda for his warm hospitality and the Japan Society for the Promotion of Science for its financial support. This work was started when both of the authors visited the Physics Department of Universität München as the Alexander von Humboldt research fellows.

\section{References}

[1] C.D. Froggatt and H.B. Nielsen, Nucl. Phys. B 147 (1979) 277;

S. Dimopoulos, Phys. Lett. B 129 (1983) 417;

M. Leurer, Y. Nir and N. Seiberg, Nucl. Phys. B 398 (1993) 319;

Nucl. Phys. B 420 (1994) 468;

Y. Nir and N. Seiberg, Phys. Lett. B 309 (1993) 337;

P. Binétruy and P. Ramond, Phys. Lett. B 350 (1995) 49;

E. Dudas, S. Pokorski and C.A. Savoy, Phys. Lett. B 356 (1995) 45. 
[2] R. Ramond, R.G. Roberts and G.G. Ross, Nucl. Phys. B 406 (1993) 19.

[3] L.E. Ibáñez and G.G. Ross, Phys. Lett. B 332 (1994) 100.

[4] M. Drees, Phys. Lett. B 181 (1986) 279;

J.S. Hagelin and S. Kelley, Nucl. Phys. B 342 (1990) 95;

A.E. Faraggi, J.S. Hagelin, S. Kelley and D.V. Nanopoulos, Phys. Rev. D 45 (1992) 3272;

Y. Kawamura, Phys. Rev. D 53 (1996) 3779.

[5] J. Ellis and D.V. Nanopoulos, Phys. Lett. B 110 (1982) 44;

R. Barbieri and R. Gatto, Phys. Lett. B 110 (1982) 211;

T. Inami and C.S. Lim, Nucl. Phys. B 207 (1982) 533;

J. Hagelin, S. Kelly and T. Tanaka, Nucl. Phys. B 415 (1994) 293.

[6] T. Kobayashi,Phys. Lett. B 354 (1995) 264.

[7] L. Dixon, J. Harvey, C. Vafa and E. Witten, Nucl. Phys. B 261 (1985) 678; Nucl. Phys. B 274 (1986) 285;

L.E. Ibáñez, J. Mas, H.P. Nilles and F. Quevedo, Nucl. Phys. B 301 (1988) 157;

Y. Katsuki, Y. Kawamura, T. Kobayashi, N. Ohtsubo, Y. Ono and K. Tanioka, Nucl. Phys. B 341 (1990) 611.

[8] T. Kobayashi, Phys. Lett. B 358 (1995) 253.

[9] A.E. Faraggi and E. Halyo, Nucl. Phys. B 416 (1994) 63;

N. Haba, C. Hattori, M. Matsuda, and T. Matsuoka, Preprint DPNU-96-23, AUE-96/02, hep-ph/9605238.

[10] Y. Kawamura and T. Kobayashi, Phys. Lett. B 375 (1996) 141; Preprint DPSU-96-11, INS-Rep-1153, hep-ph/9608233;

Y. Kawamura, T. Kobayashi and T. Komatsu, Preprint DPSU-96-12, INS-Rep-1161, hep$\mathrm{ph} / 9609462$.

[11] S. Hamidi and C. Vafa, Nucl. Phys. B 279 (1987) 465;

L. Dixon, D. Friedan, E. Martinec and S. Shenker, Ncul. Phys. B 282 (1987) 13.

[12] T. Kobayashi and N. Ohtsubo, Phys. Lett. B 245 (1990) 441; Int. J. Mod. Phys. A 9 (1994) 87.

[13] M. Cvetic, Phys. Rev. Lett. 59 (1987) 2829;

A. Font, L.E. Ibáñez, H.P. Nilles and F. Quevedo, Phys. Lett. B 213 (1988) 274. 
[14] J. Erler, D. Jungnickel and J. Lauer, Phys. Rev. D 45 (1992) 3651;

D. Jungnickel, J. Lauer, M. Spaliński and S. Stieberger, Mod. Phys. Lett. A 7 (1992) 3059;

J. Erler, D. Jungnickel, M. Spaliński and S. Stieberger, Nucl. Phys. B 397 (1993) 379.

[15] C.S. Lim, Phys. Lett. B 256 (1991) 233;

M. Dine R.G. Leigh and D.A. MacIntire, Phys. Rev. Lett. 69 (1992) 2030;

K. Choi, D.B. Kaplan and A.E. Nelson, Nucl. Phys. B 391 (1993) 515;

T. Kobayashi and C.S. Lim, Phys. Lett. B 343 (1995) 122.

[16] H. Fritzsch, Phys. Lett. B 73 (1978) 317; Nucl. Phys. B 155 (1979) 189.

[17] H. Fritzsch and Z.Z. Xing, Phys. Lett. B 353 (1995) 114.

[18] K.S. Babu and Q. Shafi, Phys. Rev. D 47 (1993) 5004; and references therein.

[19] Z.Z. Xing, Nucl. Phys. B (Proc. Suppl.) 50 (1996) 24; Nuovo Cimento A 109 (1996) 115.

[20] Particle Data Group, R.M. Barnett et al., Phys. Rev. D 54 (1996) 1.

[21] H. Leutwyler, Phys. Lett. B 378 (1996) 313.

[22] A. Ali and D. London, Preprint DESY 96-140, UdeM-GPP-TH-96-38.

[23] See, e.g., L.J. Hall and A. Rasin, Phys. Lett. B 315 (1993) 164;

D. Du and Z.Z. Xing, Phys. Rev. D 48 (1993) 2349.

[24] K. Harayama, N. Okamura, A.I. Sanda, and Z.Z. Xing, Preprint DPNU-96-36, hepph/9607461; and references therein;

K. Harayama and N. Okamura, Preprint DPNU-96-24 (to appear in Phys. Lett. B).

[25] Z.Z. Xing, Preprint DPNU-96-39, hep-ph/9609204.

[26] See, e.g., G.C. Branco, D. Emmanuel-Costa, and J.I. Silva-Marcos, Preprint hep$\mathrm{ph} / 9608477$;

K. Wang, Preprint UCLA/96/TEP/14, hep-ph/9605237.

For earlier and more extensive references, see: C.D. Froggatt, Preprint GUTPA/96/02/1, hep-ph/9603432.

[27] J. Gasser and H. Leutwyler, Phys. Rep. 87 (1982) 77.

[28] M. Neubert, Int. J. Mod. Phys. A 11 (1996) 4173.

[29] H. Georgi and C. Jarlskog, Phys. Lett. B 86 (1979) 297. 


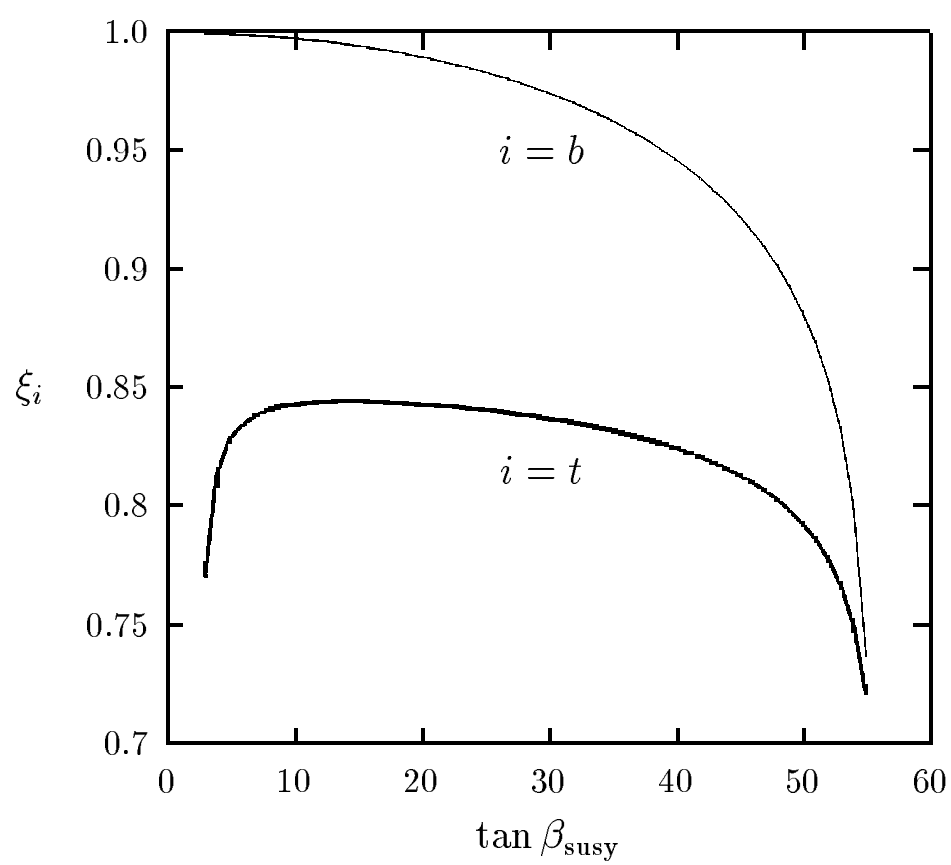

Figure 1: The magnitudes of $\xi_{t}$ and $\xi_{b}$ changing with $\tan \beta_{\text {susy }}$ in the MSSM.

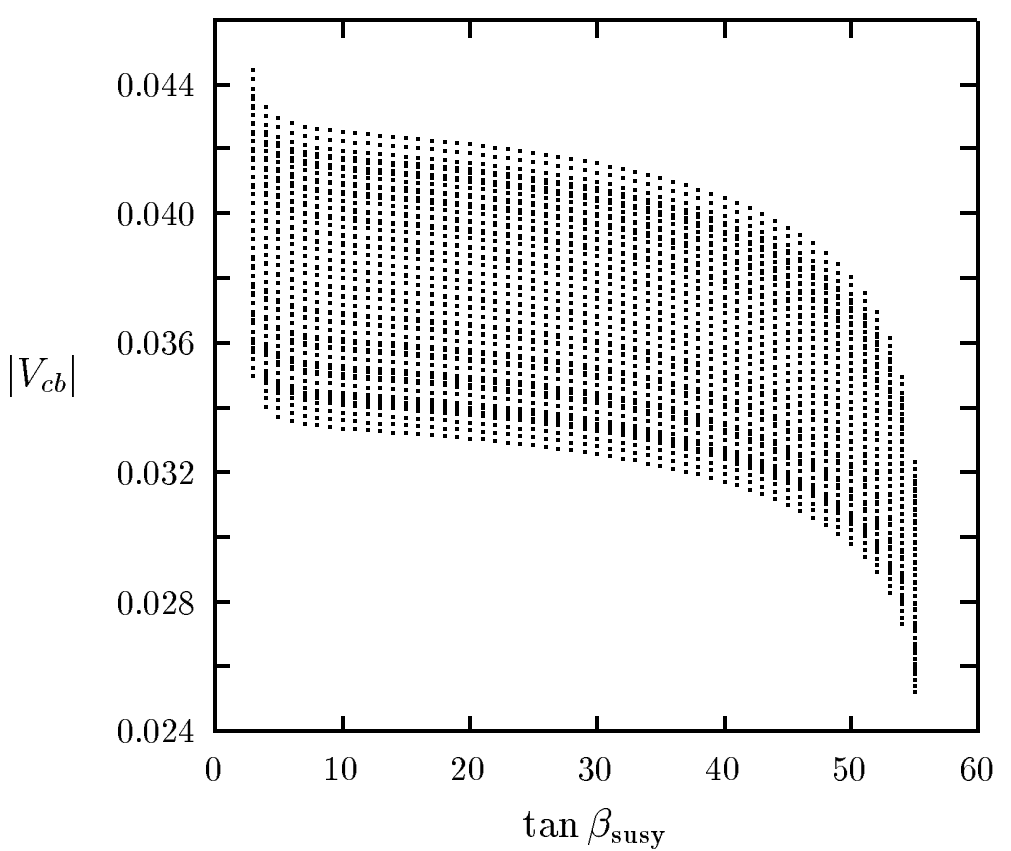

Figure 2: The allowed region of $\left|V_{c b}\right|$ (as a function of $\tan \beta_{\text {susy }}$ ) at the weak scale $M_{Z}$. 